\title{
A Quick Assessment and Optimization Method for a Flutter Aerodynamic Measure of a Typical Flat Box Girder
}

\author{
Feng Wang $\mathbb{D}^{1}{ }^{1}$ Chuan Xiong $\mathbb{D}^{1},{ }^{1}$ Zijian Wang, ${ }^{1}$ Congmin Guo, ${ }^{2}$ Hua Bai, ${ }^{1}$ and Jiawu Li ${ }^{1}$ \\ ${ }^{1}$ School of Highway, Chang'an University, Xi'an 710000, China \\ ${ }^{2}$ CCCC First Highway Consultants Co.,Ltd., Xi'an 710075, China
}

Correspondence should be addressed to Chuan Xiong; 2018121023@chd.edu.cn

Received 4 June 2020; Revised 26 July 2020; Accepted 28 July 2020; Published 12 August 2020

Academic Editor: Evgeny Petrov

Copyright (c) 2020 Feng Wang et al. This is an open access article distributed under the Creative Commons Attribution License, which permits unrestricted use, distribution, and reproduction in any medium, provided the original work is properly cited.

Flutter is one of the most serious wind-induced vibration phenomena for long-span bridges and may cause the collapse of a bridge (e.g., the Old Tacoma Bridge, 1940). The selection and optimization of flutter aerodynamic measures are difficult in wind tunnel tests. It usually takes a long time and consumes more experimental materials. This paper presents a quick assessment and design optimization method for the flutter stability of a typical flat box girder of the long-span bridges. Numerical analysis could provide a reference for wind tunnel tests and improve the efficiency of the test process. Based on the modal energy exchange in the flutter microvibration process, the global energy input and local energy input are analyzed to investigate the vibration suppression mechanism of a flat steel box girder with an upper central stabilizer. Based on the comparison between the experimental and numerical data, a quick assessment method for the optimization work is proposed. It is practical to predict the effects of flutter suppression measures by numerical analysis. Thus, a wind tunnel test procedure for flutter aerodynamic measures is proposed which could save time and experimental materials.

\section{Introduction}

As the spans of bridges have become larger, the bridge deck section of long-span bridges suffers aeroelastic phenomena of flutter and buffeting, as well as VIV or galloping, and the aerodynamic stability requirements of long-span bridges have become greater [1]. Flutter is one of the most serious wind-induced vibration phenomena for bridges, which causes the entire structure to fail. Researchers have carried out many studies to investigate the mechanism of flutter such as identification of flutter derivatives and the flutter aerodynamic measures $[2,3]$.

Based on the coupling effect of aerodynamic forces, Yang et al. [4-6] established the relationship between torsional, vertical, and lateral vibration parameters and flutter derivatives (e.g., using the two-dimensional three-degree-of-freedom coupled flutter analysis method) and studied the flutter mechanism of two typical bridge sections: the closed box girder and two-isolated-girder sections. The flutter of these two sections is caused by negative aerodynamic damping, and the negative aerodynamic damping of the streamlined section is the result of the coupling effect of torsion and vertical motion. With the rapid development of CFD (computational fluid dynamics) methods [7], numerical simulation technology has been applied in the field of bridge wind engineering [8-10]. Vairo [11] used the RNG turbulence model to simulate the self-excited force acting on the typical main beam section. The identified flutter derivatives are basically consistent with the wind tunnel test results. Shirai and Ueda [12] used a nonlinear turbulence model $k-\varepsilon$ to identify the flutter derivatives of the slotted section and the section with the central stabilizer, and the flutter mechanism was explained by its calculated flow field morphology and unsteady wind pressure distribution. Sarwar et al. [13] used the LES turbulence model to investigate the aerodynamic characteristics of a box girder bridge section. The flow around the streamline box girder section was analyzed, and the effect of section details on the aerodynamic characteristics of the bridge section was evaluated. Sun et al. [14] explored the turbulence model for the numerical calculation of fluid-solid coupling 
and calculated 18 flutter derivatives of the rectangular section with aspect ratios of $B / D=4$ using the standard $k-\omega$ model; in this way, the influence of inflow turbulence intensity on the flutter derivatives was studied. Bai et al. [15] proposed an improved CFD method based on block-iterative coupling, performing $2 \mathrm{D}$ and $3 \mathrm{D}$ CFD simulations on two generic bridge deck sections to determine their aerodynamic force coefficients and flutter derivatives. Šarkić et al. [16] used the SST $k-\omega$ turbulence model to identify the flutter derivatives of a streamlined section, and their results are in good agreement with the experimental data.

In order to investigate the mechanism of the occurrence of flutter and the mechanism of vibration suppression by aerodynamic measures, many scholars have focused on energies and pressures. Scanlan [17] proposed a multimode flutter analysis technique from the viewpoint of the energy of the system. Based on the motion law of the vortex in discrete vortex calculation, Larsen [18] proposed a simplified analysis model that describes the motion of the vortex of the bridge section during a torsional motion cycle and estimates the total work done via the aerodynamic force generated by the vortex on the bridge section; the model then estimates the critical wind speed of the flutter. Chen et al. [19] studied the mechanism of multimodal coupled flutter from the perspective of system energy. Liu et al. [20] combined structure and airflow as a system to establish a new calculation method for the flutter analysis of suspension bridges from the perspective of energy balance. Liu et al. [21] used CFD to simulate the flutter process of the box girder and gave a definition for the aerodynamic energy calculation formula of the airflow input to the vibration model and the aerodynamic energy ratio of the surface area of the model. The flutter stability criterion was given from the viewpoint of energy balance. Yang et al. [22] used a method of flutter energy analysis to investigate the components of energy input and energy dissipation, and their results showed that components associated with flutter derivatives $A_{1}^{*}$ and $A_{2}^{*}$ produce the maximum positive and negative energy inputs, respectively. The maximum energy dissipation has a strong correlation with mechanical damping force. Tang et al. [23] studied the effects of different horizontal and vertical aerodynamic countermeasures, and flutter performance was judged by calculating the input energy by aerodynamic forces.

Although scholars have performed many wind tunnel tests and numerical simulations in the study of flutter mechanisms and vibration suppression measures, the selection and optimization of flutter aerodynamic measures are difficult to obtain in wind tunnel tests. In this study, the wind tunnel test method is used to study the energy input of the vibration suppression mechanism of the upper central stabilizer. The characteristics of global energy input and local energy input were investigated, which together demonstrate the mechanism from a macroscopic and microcosmic perspective. Then, the numerical simulation method is used to accomplish bending-torsion coupling flutter for the box girder, and a numerical analysis of the effect of the upper central stabilizer on flutter stability was conducted, so the numerical simulation method can be applied to the subject, and the obtained result is determined to be applicable.

\section{Wind Tunnel Test Setup and Data Processing Method}

2.1. Experimental Models. In order to study the effect of the upper central stabilizer, a closed flat steel box girder with an aspect ratio of $11: 1$ was taken as the prototype in the wind tunnel test. According to the requirements of the wind tunnel test, the scale ratio of the sectional model is $1: 75$, the model length $L$ is $2 \mathrm{~m}$, the width $B$ is $0.596 \mathrm{~m}$, and the height $H$ is $0.054 \mathrm{~m}$. Three kinds of test conditions were proposed, which were the original section and the original section with the upper central stabilizer with the height of $0.3 \mathrm{H}$ or $0.4 \mathrm{H}$. Figure 1 shows the schematic diagram of the models. Meanwhile, 102 pressure taps were arranged at the middle section of the models. The schematic diagram of the pressure measuring point of the model is shown in Figure 2.

The skeleton of the model was welded by aluminum alloy tubes. The surface of the model was made from aerospace wood and electrical boards. The edges of the model were equipped with end plates made of acrylic. A picture of the sectional model for the construction stage is shown in Figure 3.

For the simulation of aeroelastic parameters in the wind tunnel test, a first-order symmetrical vertical bending mode and a first-order symmetric torsional mode were simulated for the girder of a long-span suspension bridge. Table 1 shows the model's design parameters.

2.2. Wind Tunnel Test Procedure. The wind test was carried out in the CA-01 wind tunnel at Chang'an University. The test section was $15.0 \mathrm{~m}$ long, and the cross section was $3.0 \mathrm{~m}$ wide and $2.5 \mathrm{~m}$ high; the adjustable wind speed ranged from 0.2 to $53 \mathrm{~m} / \mathrm{s}$, and the turbulence of the uniform field was less than $0.3 \%$. The wind tunnel test included synchronous vibration measurement, force measurement, and pressure measurement. In the free vibration tests (2-dof only-vertical and torsional), two laser displacement meters were installed at each end of the model to measure the displacement. During aeroelastic vibration, the electronic pressure scanner valves made by PSI were used to obtain the pressure, and the sampling rate is $312.5 \mathrm{~Hz}$, as shown in Figure 4 . In the aerodynamic force tests, the strain-gauge balance was used when the sectional model was fixed. Through the vibration test, the effects of different upper central stabilizers on the bending-torsion coupling flutter were measured. Through the wind force test, the aerodynamic force coefficients of the girder were obtained. Moreover, the wind pressure distribution on the surface of the model was measured synchronously to study the influence of different aerodynamic measures on the surface pressure distribution.

2.3. Data Processing Method. The mean aerodynamic force coefficients are nondimensional parameters describing the magnitude of the static wind loads acting on the structure. Through a force test of the sectional model, this paper measured the drag force coefficient $C_{D}$, the lift force coefficient $C_{L}$, and the torque coefficient $C_{M}$ of each static model with different aerodynamic measures, which are defined as follows: 


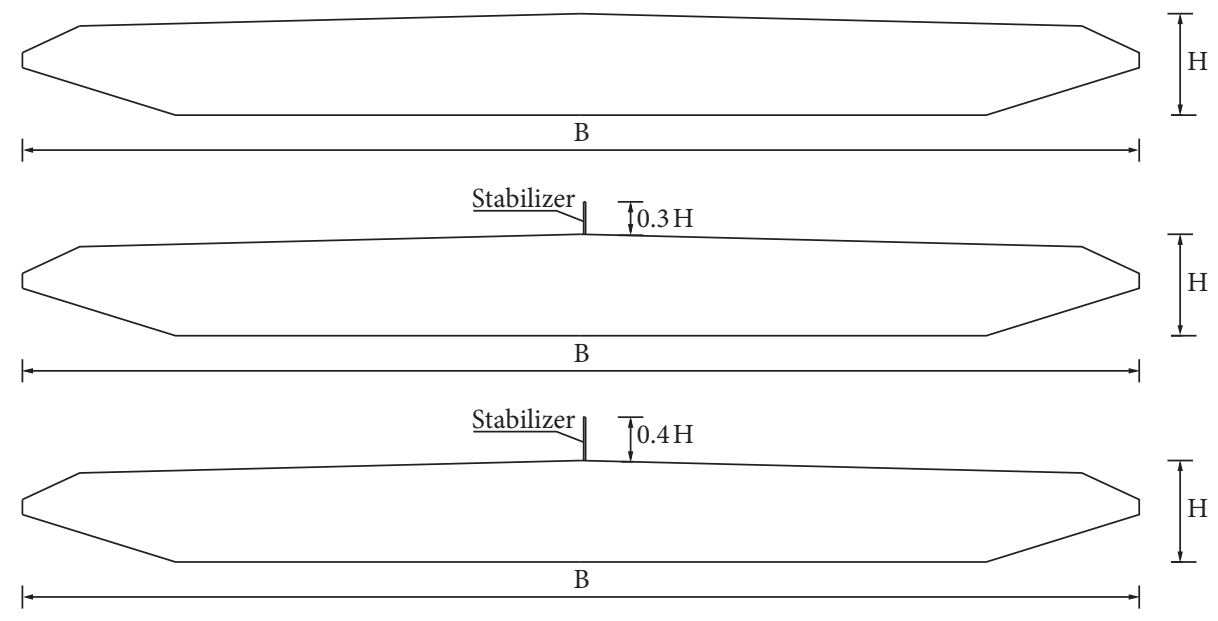

Figure 1: Experimental models.

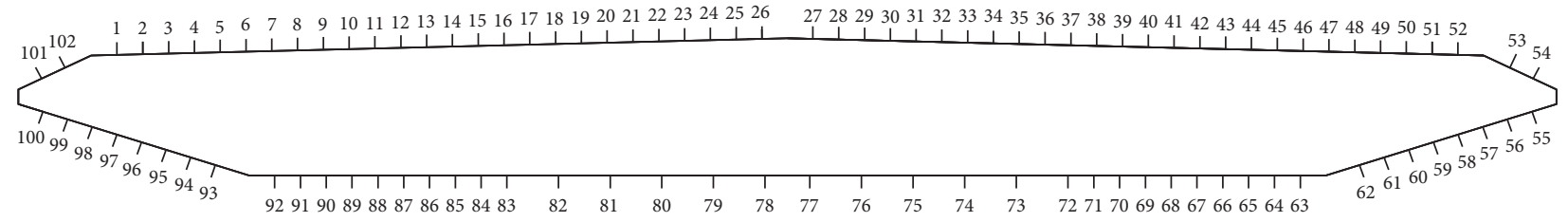

FIgURE 2: Arrangement of pressure taps.

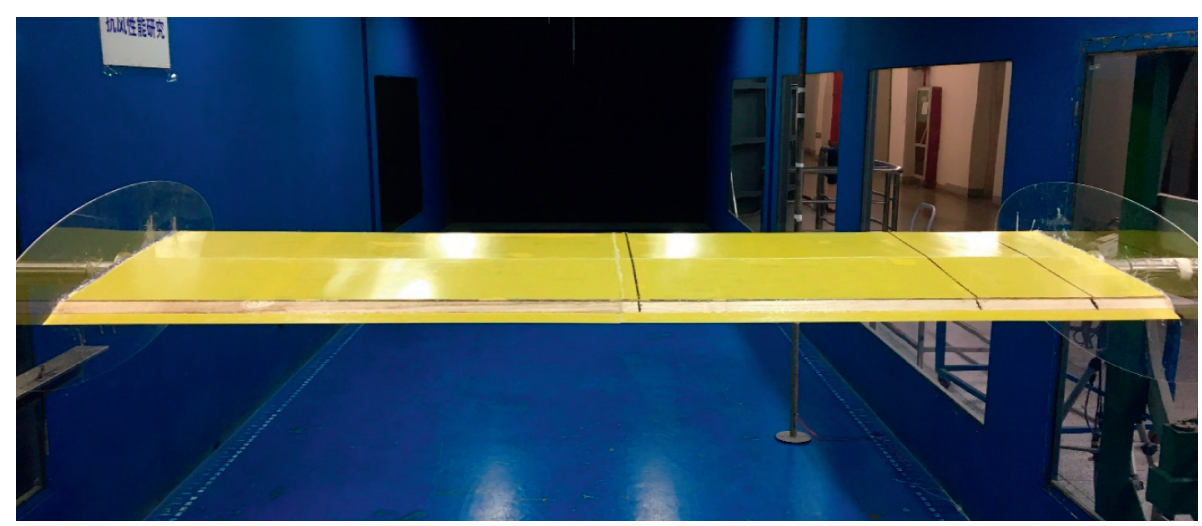

FIgURE 3: Experimental model in the wind tunnel test.

$$
\begin{aligned}
C_{D} & =\frac{F_{D}}{(1 / 2) \rho U^{2} D L}, \\
C_{L} & =\frac{F_{L}}{(1 / 2) \rho U^{2} B L}, \\
C_{M} & =\frac{M}{(1 / 2) \rho U^{2} B^{2} L},
\end{aligned}
$$

where $F_{D}, F_{L}$, and $M$ are the drag, lift, and torque of the sectional girder model, respectively, $\rho$ is the air density, $U$ is the mean longitudinal wind speed, and $B$ and $L$ are the height and width of the sectional girder model, respectively.

The wind pressures on the models are expressed in the form of a nondimensional pressure coefficient, defined as

$$
C_{P}(i, t)=\frac{C_{P}(i, t)-P_{0}}{(1 / 2) \rho U^{2}},
$$

where $C_{P}$ is the wind pressure coefficient at tap $i$ and time $t$ on the outer surface of the model and $P_{0}$ is the static reference pressure.

\section{Wind Tunnel Test Results}

3.1. Static Aerodynamic Force Coefficients. Figures 5 and 6 show a comparison of the mean aerodynamic force coefficients between the original section and the section with central stabilizers at heights of $0.3 \mathrm{H}$ and $0.4 \mathrm{H}$. It can be seen that, at $0^{\circ}$ (the wind attack angle), both the original section and the section with the central stabilizer are subjected to a downwardly negative lift force, and the absolute value of the 
TABLE 1: Design parameters of the model of the original girder section.

\begin{tabular}{lccc}
\hline Parameters & Symbol & Unit & Measured value \\
\hline Equivalent mass & $m$ & $\mathrm{~kg} / \mathrm{m}$ & 15.080 \\
Equivalent inertia movement & $J_{m}$ & $\mathrm{~kg} \cdot \mathrm{m}^{2} / \mathrm{m}$ & 0.622 \\
Fundamental vertical bending frequency & $f_{v}$ & $\mathrm{~Hz}$ & 1.274 \\
Fundamental torsional frequency & $f_{t}$ & $\mathrm{~Hz}$ & 2.839 \\
Vertical bending damping ratio & $\zeta_{v}$ & - & $6.7 \%$ \\
Torsional damping ratio & $\zeta_{t}$ & - & $3.5 \%$ \\
\hline
\end{tabular}

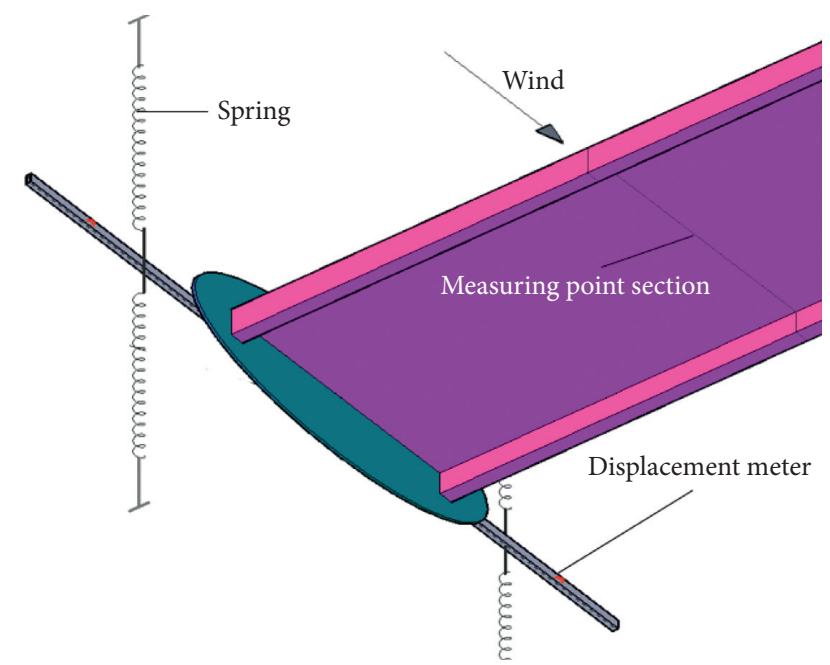

FIGURE 4: Schematic diagram of the synchronous measurement system.

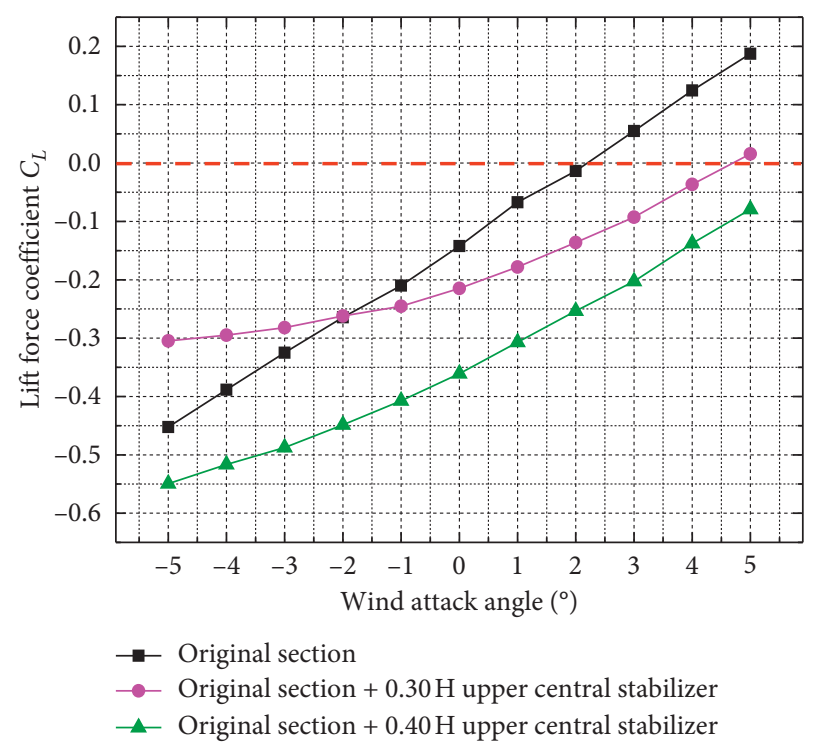

Figure 5: Lift force coefficients for different wind attack angles.

negative lift gradually increases as the height of the upper central stabilizer increases. To study the relationship between the lift force coefficient and the flutter stability, the zero curve of the lift coefficient is illustrated in Figure 4. Considering the relationship between the vibrational amplitude and the wind speed, the earlier the lift coefficient curve intersects the zero curve, the more easily the model is subjected to the positive lift during microvibrations and the less the flutter stability. It can

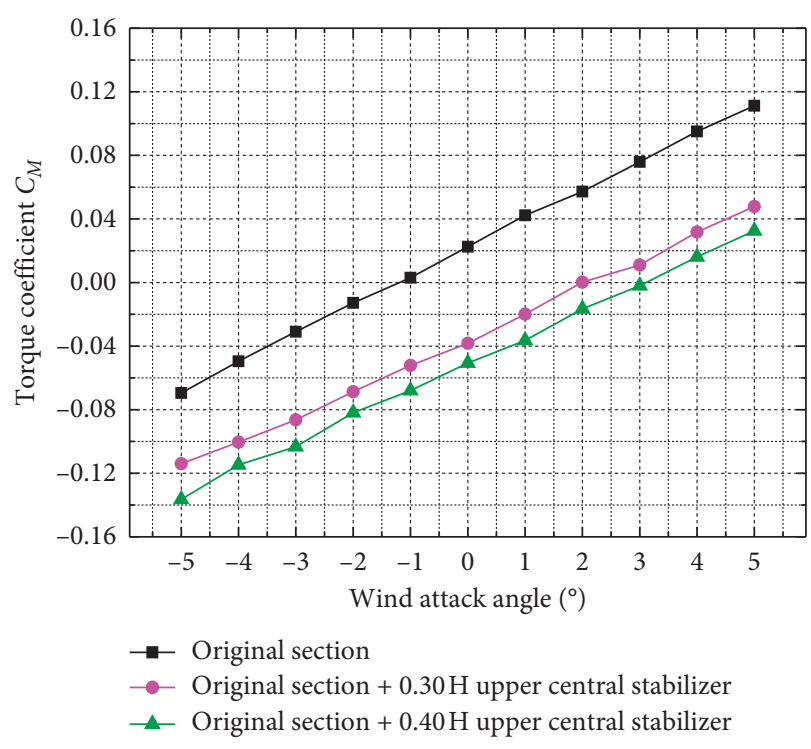

Figure 6: Torque coefficients for different wind attack angles.

be seen in Figure 4 that the flutter stability of the original section is poor, and the flutter stability of the central stabilizer with a height of $0.4 \mathrm{H}$ is the best. On the contrary, the values of the torque coefficient decrease as the height of the upper central stabilizer increases.

3.2. Relationship between Amplitude and Wind Speed. Based on the relationship between vibration amplitude and wind speed, it can be judged whether flutter occurred, and the critical wind speed of the flutter can be determined. Figures 7 and 8 show a comparison of the vibration response of the model sections with different central stabilizers. The vibration responses in the figures are converted into the root mean square (RMS) value of the full-scale vertical displacement and torsional angle. It is found that the flutter occurs in the original section at a $0^{\circ}$ wind attack angle, and the critical wind speed of the flutter is near $14.1 \mathrm{~m} / \mathrm{s}$. When the central stabilizer with a height of $0.3 \mathrm{H}$ or $0.4 \mathrm{H}$ is applied, the flutter can be suppressed.

\subsection{Pressure Distribution Characteristics Acting on the Model} Surfaces. Figures 9 and 10 show the mean wind pressure coefficient acting on the upper and lower surfaces of the original section and the section with the upper central stabilizers, where $X_{p}$ is the lateral distance from each measuring point to the center of the model. It can be found 


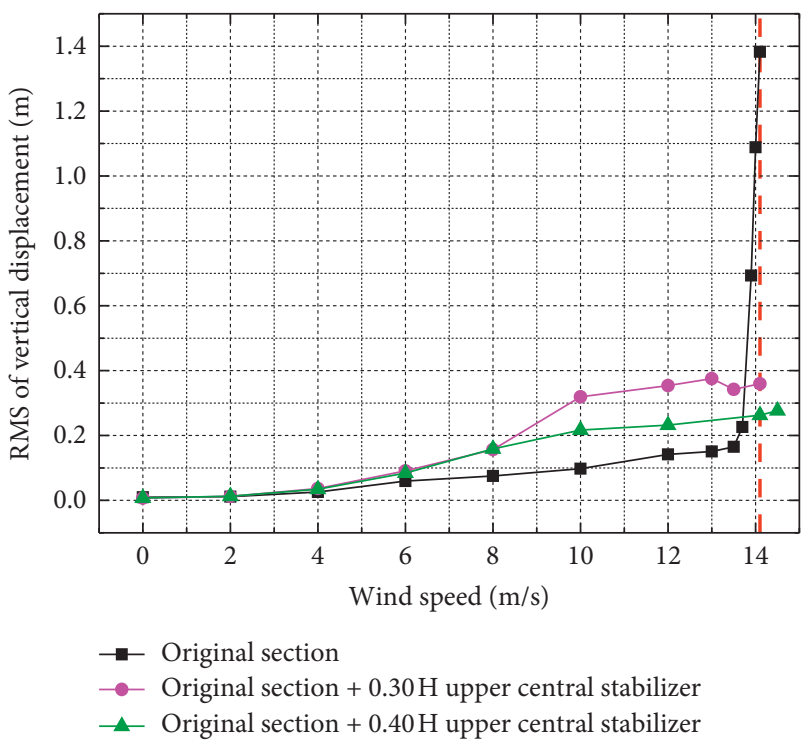

Figure 7: Vertical displacements for different aerodynamic measures.

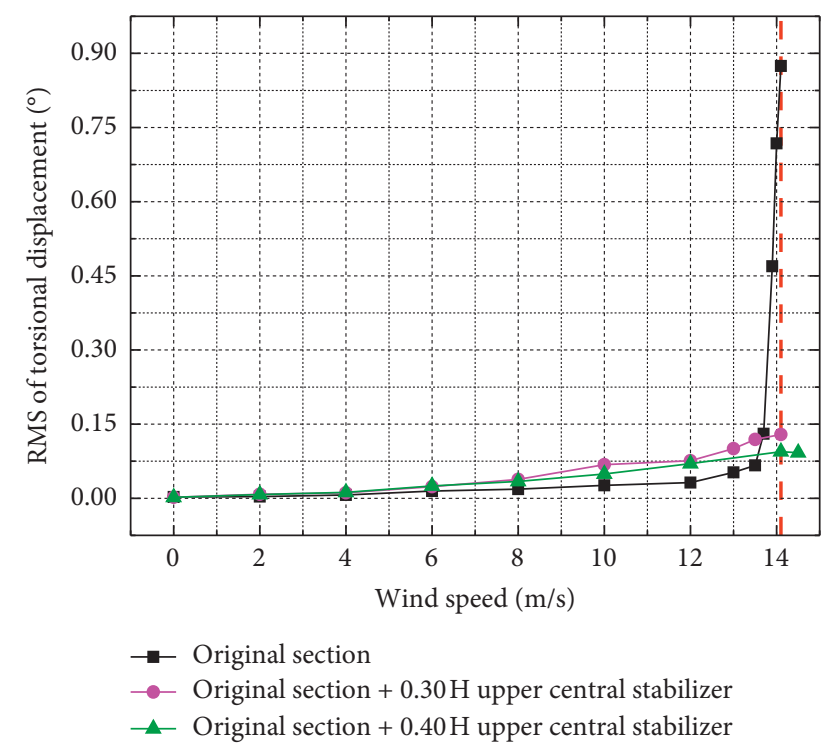

Figure 8: Torsional displacements for different aerodynamic measures.

that most pressure taps of the original model's surface are under negative pressure. When the central stabilizes are with heights of $0.3 \mathrm{H}$ and $0.4 \mathrm{H}$, positive pressure appears in the upstream region of the upper surface of the girder section, and the mean wind pressure coefficient in the downstream area reduces greatly. As a result, the lift force upstream from the upper surface changes from a negative lift force to a positive lift force, and the lift force in the downstream region changes from small negative values into larger values, which inevitably lead to a negative torque for the model. For the lower surface of the model, the wind pressure distribution trend is almost the same before and after the upper central stabilizer is applied.

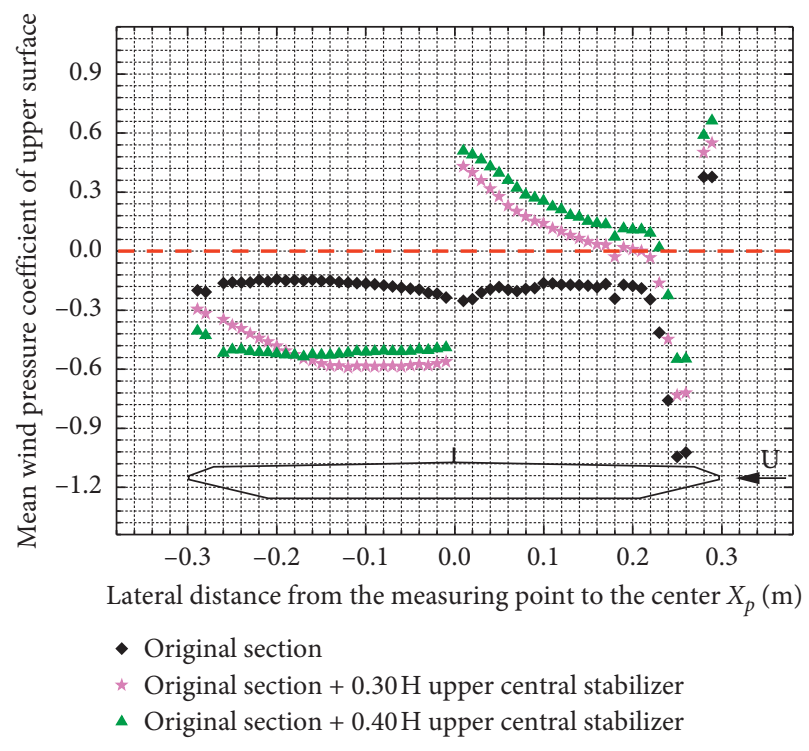

FIGURE 9: Mean wind pressure coefficients of upper surfaces.

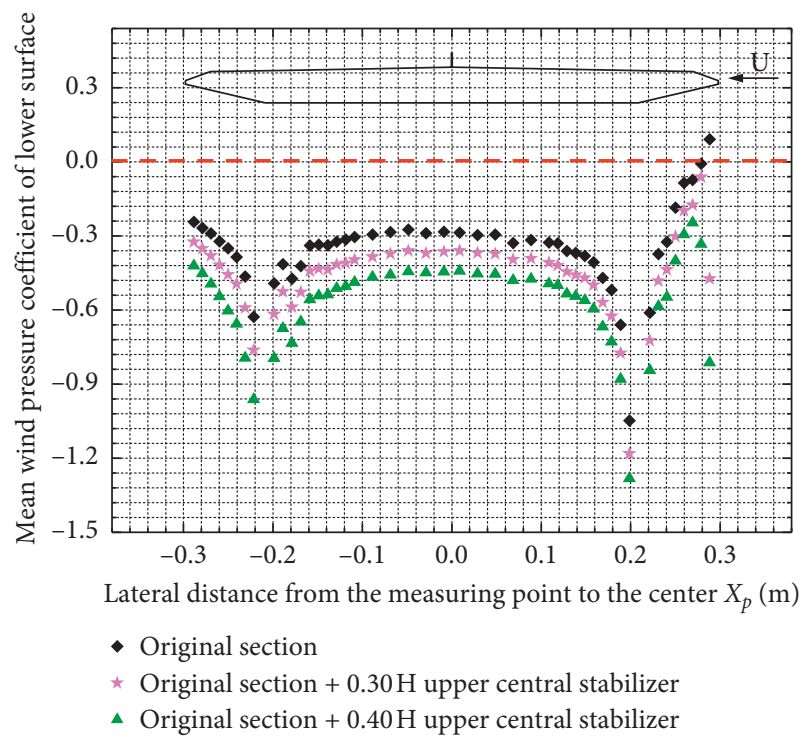

Figure 10: Mean wind pressure coefficients of lower surfaces.

However, as the height of the upper central stabilizer increases, the absolute value of the mean wind pressure coefficient increases slightly, which causes the negative lift force of the lower model surface to increase.

\section{Energy Input Characteristics of the Upper Central Stabilizer}

\subsection{Global Energy Input Characteristics}

\subsubsection{Calculation Method}

(1) Motion Equation of the Vibration System. In order to simplify the analysis, this study only considers a two-degreeof-freedom bending-torsion coupling vibration system. 
During the critical state of the flutter, the vertical displacement and torsional displacement of the vibration system, during a short-term vibration period, are regarded as the simple harmonic vibrations of different phases at the same frequency, which are expressed as follows:

$$
\begin{aligned}
& h(t)=h_{0} \sin \left(\omega t+\varphi_{1}\right), \\
& a(t)=a_{0} \sin \left(\omega t+\varphi_{2}\right),
\end{aligned}
$$

where $h_{0}$ and $a_{0}$ are the vertical bending amplitude and the torsional amplitude, $\omega$ is the vibration circular frequency when the flutter occurs, and $\varphi_{1}$ and $\varphi_{2}$ are the initial phases of the vertical displacement and the torsional displacement.

(2) Time History of Global Aerodynamic Force. In this study, the time history of global self-excited aerodynamic force is obtained by full-section pressure integration. Using MAT$\mathrm{LAB}$ programming to perform the integration process for pressures at several moments in several vibration cycles, the aerodynamic time history $F_{L}(t)$ and $M(t)$ can be obtained to calculate the aerodynamic work.

(3) Global Aerodynamic Force Doing Work. For the twodegree-of-freedom bending-torsion coupling vibration system, if the calculation time $t_{0}$ is selected as a reference point, when the section vibrates until time $t_{1}$, the cumulative work of the self-excited aerodynamic force for the vertical and torsional degrees of freedom is

$$
\left\{\begin{array}{l}
W_{s e}^{H}\left(t_{1}\right)=\int_{t_{0}}^{t_{1}} F_{L}(t) \dot{h}(t) \mathrm{d} t, \\
W_{s e}^{T}\left(t_{1}\right)=\int_{t_{0}}^{t_{1}} M(t) \dot{h}(t) \mathrm{d} t
\end{array}\right.
$$

where $W_{s e}^{H}\left(t_{1}\right)$ and $W_{s e}^{T}\left(t_{1}\right)$ are the work done by the selfexcited aerodynamic force on the vertical and torsional degrees of freedom, respectively, and $F_{L}(t)$ and $M(t)$ are the lift force and torque time history, respectively.

Thus, the total work done by the self-excited aerodynamic force in the vibration system is

$$
W_{s e}\left(t_{1}\right)=W_{s e}^{H}\left(t_{1}\right)+W_{s e}^{T}\left(t_{1}\right)
$$

(4) Mechanical Energy Increment. In the fluid-solid coupling system, the vibration system is not conservative, and the mechanical energy of the system is not a constant. The work done by the aerodynamic force is partially dissipated by structural damping, and the other portion is converted into the mechanical energy of the system. If one vibration period is considered, the mechanical energy increment of the system can be expressed as

$$
\Delta E_{M}=W_{s e}+W_{D}
$$

where $W_{s e}$ and $W_{D}$ are the work done by the self-excited aerodynamic force and structural damping, respectively.

(5) Criterion for the Effectiveness of Flutter Aerodynamic Measures Based on the Energy Method. From the perspective of energy balance, while the vibration system is in a complete vibration cycle, the mechanical energy increment $\Delta E_{M}$ is greater than 0 . If the sum of the aerodynamic work and the structural damping dissipative work is greater than 0 , the flutter has a tendency to be excited. When the mechanical energy increment $\Delta E_{M}$ is less than 0 , the sum of the aerodynamic work and the structural damping dissipative work is less than 0 , and the flutter has a tendency to be suppressed. Thus, when the mechanical energy increment $\Delta E_{M}$ is equal to 0 , the flutter is at a critical state. However, under normal circumstances, due to the small damping of the bridge structure, the structural damping dissipation work is also small. Thus, this phenomenon can be largely ignored. The positive or negative values of the aerodynamic cumulative work in a vibration cycle will directly determine whether the aerodynamic measures are effective.

4.1.2. Analysis of Calculation Results. Figure 11 shows a comparison of the global aerodynamic cumulative work of the section with an upper central stabilizer in a microvibration cycle, including a $0^{\circ}$ wind attack angle and a wind speed of $14.1 \mathrm{~m} / \mathrm{s}$. The cumulative work is the sum of the work, including each time step. Since the figure shows the cumulative work, the positive or negative slopes of the curve in the figure represent the positive or negative work during this period, respectively. If the slope is positive, positive work is done during this time. The positive or negative of the accumulated work value represents the input energy or the dissipated energy. Therefore, the cumulative total work at the last moment of the vibration period in this figure will be used as the criterion for the effectiveness of the aerodynamic measures based on the energy method. It can be seen from the figure that the cumulative total work of the original section at the last moment is the largest among these three girder sections. In comparison, the cumulative total work of the section with a $0.3 \mathrm{H}$ upper central stabilizer is relatively small, and the cumulative total work of the section with a $0.4 \mathrm{H}$ upper central stabilizer decreases until it is negative. Thus, it can be judged that the $0.40 \mathrm{H}$ upper central stabilizer is effective. When the cumulative total work of the section with a $0.30 \mathrm{H}$ central stabilizer shows a small positive value at the last moment and no flutter occurs during the test, it is recommended to perform a plurality of cycles to calculate the work and determine the effectiveness of the aerodynamic measures.

\subsection{Local Energy Input Characteristics}

\subsubsection{Calculation Method}

(1) Motion Equation of the Pressure Measuring Point. Based on Section 4.1.1, the displacement and velocity equations of the vertical motion and torsional motion of the section's center $O$ in a microvibration period can be obtained. The motion equation for each pressure measuring point can be calculated via the motion equation of center $O$ of the section, as shown in Figure 12. Assuming that the vertical velocity of the section's center $O$ is $v_{h}$, the torsional angular velocity is 


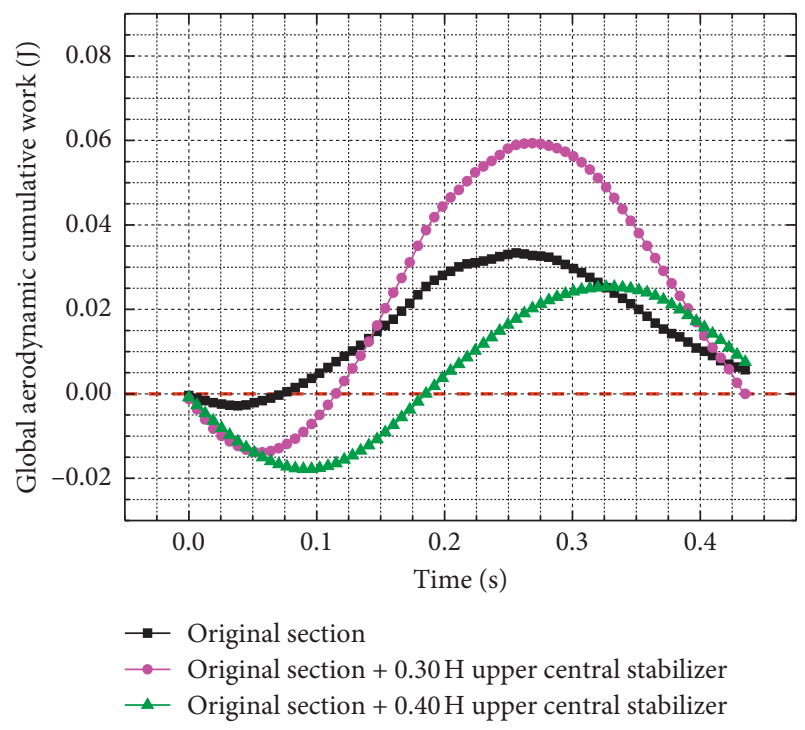

FIGURE 11: Global aerodynamic cumulative work for each time step.

$\omega$, the pressure on the microelement within the area $\mathrm{d} s_{i}$ at the $i^{\text {th }}$ pressure hole is $p_{i}$, and the distance of $p_{i}$ to the center $O$ of the section is $r$; based on the kinematics of a rigid body, the vertical velocity at the measuring point is also $v_{h}$, and the torsional linear velocity $v_{a}$ is $\omega \cdot r$.

(2) The Local Aerodynamic Force Doing Work. For the twodegree-of-freedom bending-torsion coupling vibration system, if the calculation time $t_{0}$ is selected as the reference point, when the section vibrates until time $t_{1}$, the cumulative work of the wind pressure for the local area $x$ of the section surface is

$$
\begin{aligned}
W_{(x, t)}= & \int_{t_{0}}^{t_{1}}\left[P(x, t) v_{h}(x, t) \cos \vec{P}, \vec{v}_{h}\right. \\
& \left.+P(x, t) v_{a}(x, t) \cos \vec{P}, \vec{v}_{a}\right] \mathrm{d} t,
\end{aligned}
$$

where $P(x, t)$ is the static pressure at the section's surface, $\cos \vec{P}, \vec{v}_{h}$ is the cosine of the angle between the directions that are perpendicular to the pressure tap and the direction of the vertical velocity, and $\cos \vec{P}, \vec{v}_{a}$ is the cosine of the angle between the directions that are perpendicular to the pressure tap and the direction of the torsional linear velocity.

4.2.2. Analysis of the Calculation Results. In order to compare the effects of different upper central stabilizers on the local energy input characteristics of the original section, the energy inputs acting on the upper and lower surface areas for one vibration period for different girder sections are calculated and are shown in Figures 13 and 14, respectively. It can be found that, for the upper surface of the model, the cumulative values of the original section are mostly negative, except in the downstream fairing region. The aerodynamic force dissipates energy during vibrations. In the upstream region, the cumulative work is all positive, and the self-excited aerodynamic force in this region continuously inputs energy into the vibration, which causes the original section to flutter. Once the $0.30 \mathrm{H}$ upper central stabilizer is arranged on the girder section, the cumulative work in the upstream region reduces to nearly 0 , and only the downstream region shows positive work. For the girder section with a $0.40 \mathrm{H}$ central stabilizer, the cumulative work in the upstream region reduces to a negative value. The aerodynamic force in this region continuously dissipates energy during the vibration. At the same time, compared with the $0.3 \mathrm{H}$ central stabilizer, the positive work occurring in the downstream region reduced as well, which indicates that the effect of the $0.4 \mathrm{H}$ central stabilizer is better than that of the $0.3 \mathrm{H}$ central stabilizer. The aerodynamic force in the upstream region of the upper surface of the model played a role in dissipating energy during vibration, making it the "critical region" for vibrations.

\section{Numerical Judgment of the Effectiveness of the Upper Central Stabilizer}

5.1. CFD Modeling. In order to improve the efficiency of the wind tunnel test, the effectiveness of the upper central stabilizer is studied through numerical simulation software CFD. By establishing a CFD numerical model, simulating the bending-torsion coupling vibration, verifying the accuracy of the numerical model, and calculating the energy input, the effectiveness of the measure was quantitatively judged. The length of the full flow field was about 30 times the bridge width, the width of the full flow field was more than 20 times the beam height, and the centroid of the twodimensional bridge section was located at the flow field of about $1 / 3$ the entrance. The number of mesh grids is 53,243 . The SST $k-\omega$ turbulence model is adopted. In order to better simulate the flow field at the wall's surface and the wake region of the girder section, the grid was properly encrypted at the wall and wake. The time step is 0.0032 , the spatial discretization format is second-order precision, and the SIMPLE algorithm is used. Grid meshing is shown in Figure 15 . The bending-torsion coupling vibration was realized by the UDF program driving the moving grid to realize the forced coupling vibration.

To verify the accuracy of the CFD model, the comparison of mean aerodynamic force coefficients between the wind tunnel test and numerical simulation results is listed in Table 2. This comparison shows that the numerical simulation results are close to the wind tunnel test, which explains that the CFD model is reasonable.

\subsection{Comparison of the Characteristics of Pressure Distribution.} The wind tunnel test and numerical simulation results of the pressure distribution on the upper and lower surfaces of different sections are compared in Figures 16 and 17, respectively. This comparison demonstrates that the numerical simulation results are consistent with the trend of the wind tunnel test results. The mean value of the pressure coefficient in the numerical simulation results is slightly higher than that of the wind tunnel test results. 


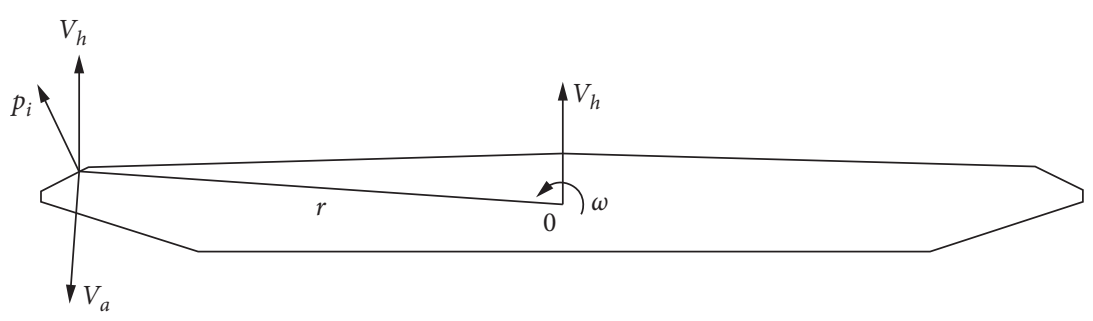

FIgURE 12: The schematic diagram of the motion equation for pressure taps.

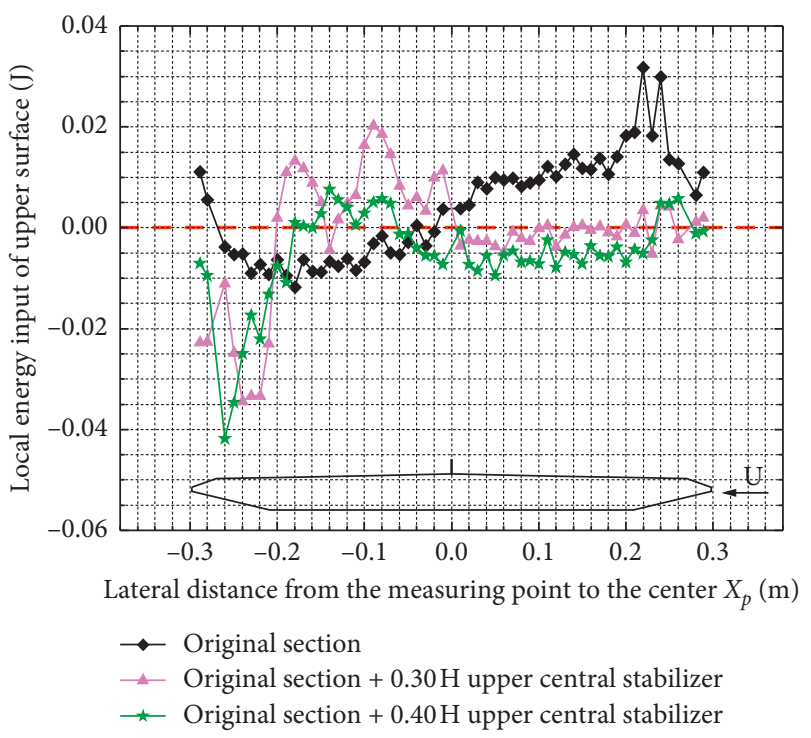

Figure 13: Local energy input of the upper surface.

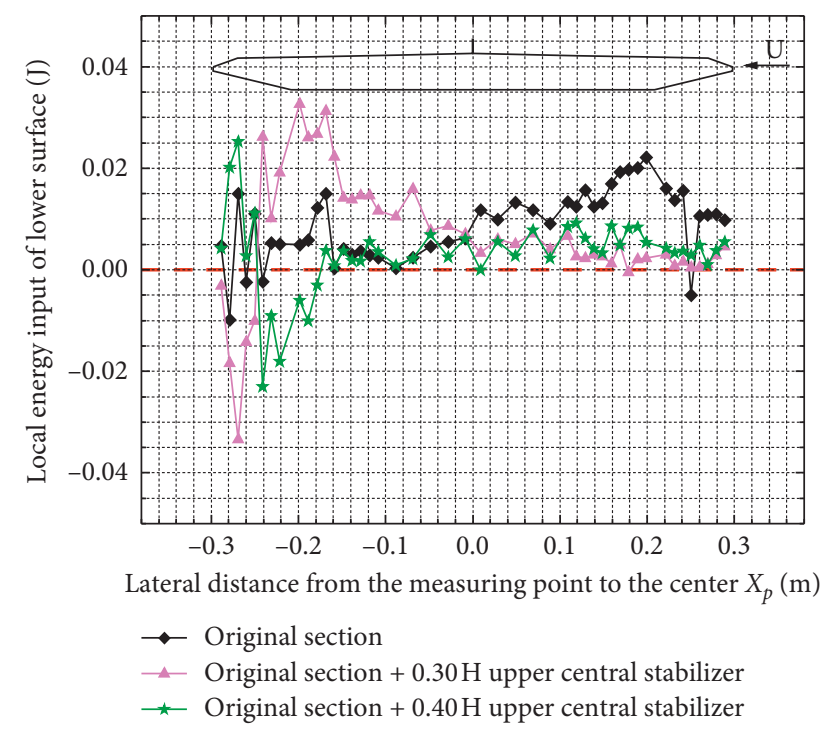

FIgURE 14: Local energy input of the lower surface.

\subsection{Numerical Judgment}

5.3.1. A Comparison of the Cumulative Global Aerodynamic Work. Through the numerical calculations in Sections 5.1 and 5.2, the lift and torque time history could be obtained. And with the calculation method mentioned in Section 4.1.1,

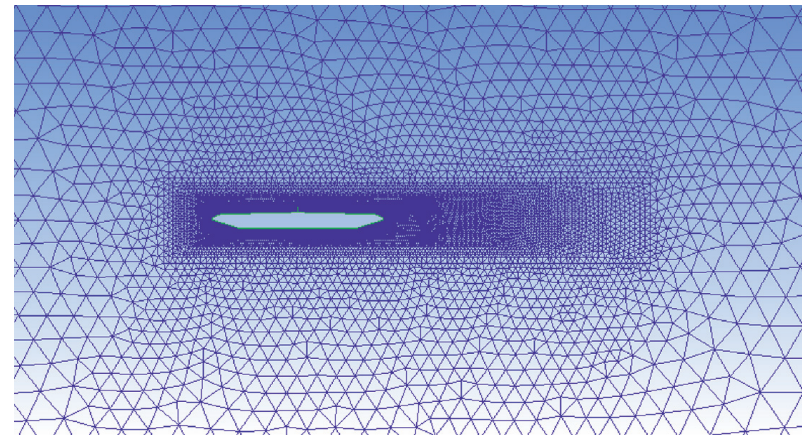

Figure 15: Meshing of the flat steel box girder (local).

TABLE 2: The mean aerodynamic force coefficients of the wind tunnel test and numerical simulation.

\begin{tabular}{lcccc}
\hline Wind attack angle & \multicolumn{2}{c}{ Wind tunnel test } & \multicolumn{2}{c}{$\begin{array}{c}\text { Numerical } \\
\text { simulation }\end{array}$} \\
& $C_{L}$ & $C_{M}$ & $C_{L}$ & $C_{M}$ \\
\hline-3 & -0.325 & -0.031 & -0.391 & -0.043 \\
0 & -0.142 & 0.042 & -0.086 & 0.010 \\
+3 & 0.055 & 0.076 & 0.108 & 0.091 \\
\hline
\end{tabular}

the cumulative global aerodynamic work in a microvibration period could be calculated.

Figure 18 shows the cumulative global aerodynamic work of the original section using upper central stabilizers with heights of $0.15 \mathrm{H}, 0.20 \mathrm{H}, 0.25 \mathrm{H}, 0.30 \mathrm{H}, 0.35 \mathrm{H}$, and $0.40 \mathrm{H}$ in a microvibration period. The wind speed is $14.1 \mathrm{~m} / \mathrm{s}$, in which flutter occurred. In order to judge the effectiveness of different upper central stabilizers, the cumulative total work at the last time step of the vibration period in this figure is compared. The cumulative total work of the section with $0.15 \mathrm{H}, 0.30 \mathrm{H}$, and $0.40 \mathrm{H}$ central stabilizers is negative, and the cumulative total work is positive for the other conditions. Thus, the numerical analysis result indicates that the central stabilizers with heights of $0.15 \mathrm{H}, 0.30 \mathrm{H}$, and $0.40 \mathrm{H}$ are effective in damping the vibrations of this girder. Moreover, comparing these three measures, the absolute value of the central stabilizer with a height of $0.4 \mathrm{H}$ is the largest. It can be judged that the damping effect of $0.4 \mathrm{H}$ is better than $0.15 \mathrm{H}$ and $0.30 \mathrm{H}$, which is consistent with the phenomenon observed in the wind tunnel test, indicating that the numerical study of the effectiveness of the upper central stabilizer has certain reliability. 


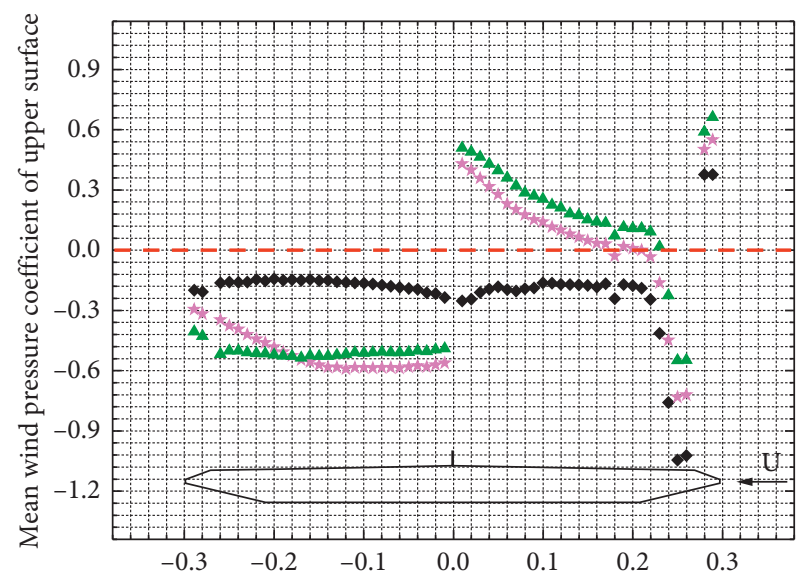

Lateral distance from the measuring point to the center $X_{p}(\mathrm{~m})$

- Original section

* Original section $+0.30 \mathrm{H}$ upper central stabilizer

^ Original section $+0.40 \mathrm{H}$ upper central stabilizer

(a)

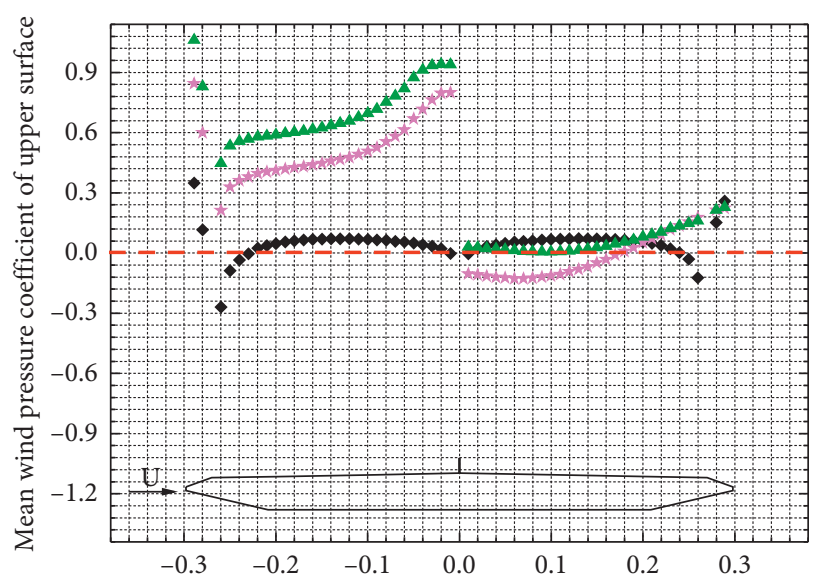

Lateral distance from the measuring point to the center $X_{p}(\mathrm{~m})$

- Original section

* Original section $+0.30 \mathrm{H}$ upper central stabilizer

^ Original section $+0.40 \mathrm{H}$ upper central stabilizer

(b)

Figure 16: Mean wind pressure coefficients of the upper surfaces of the models. (a) Wind tunnel test results. (b) Numerical simulation results.

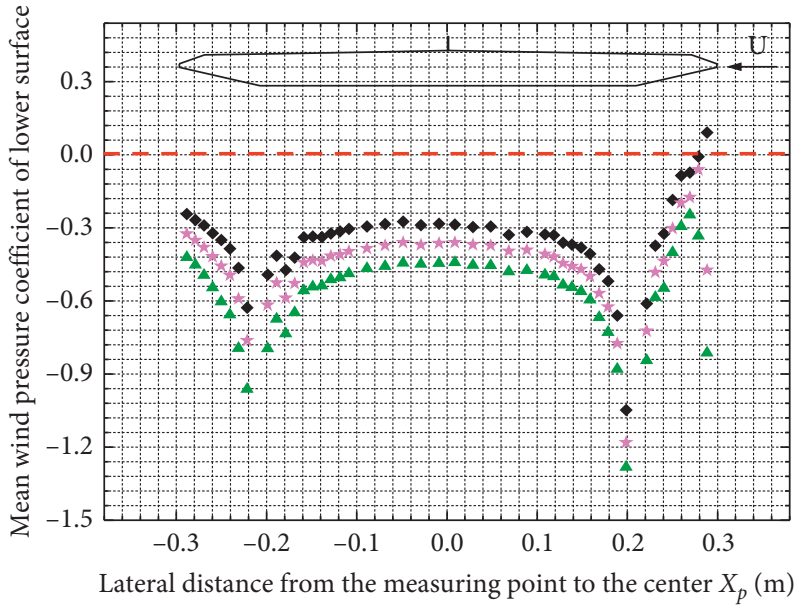

- Original section

* Original section $+0.30 \mathrm{H}$ upper central stabilizer

- Original section $+0.40 \mathrm{H}$ upper central stabilizer

(a)

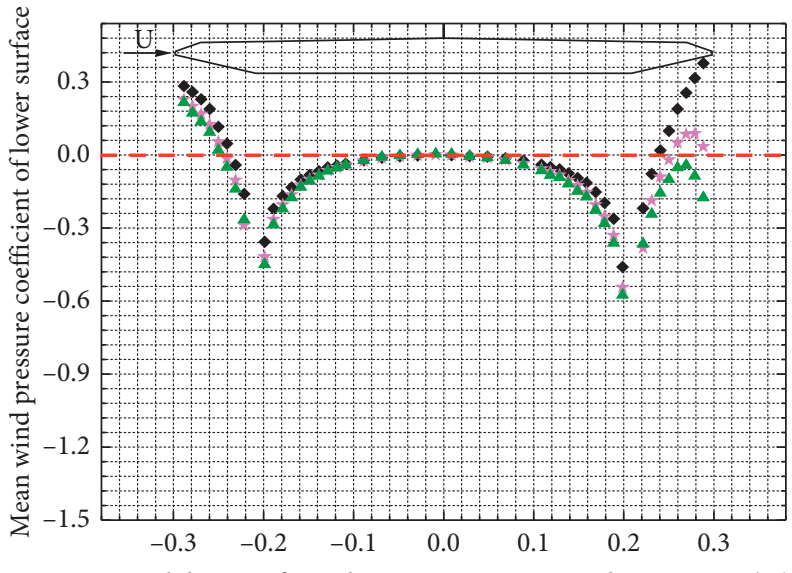

Lateral distance from the measuring point to the center $X_{p}(\mathrm{~m})$

- Original section

* Original section $+0.30 \mathrm{H}$ upper central stabilizer

^ Original section $+0.40 \mathrm{H}$ upper central stabilizer

(b)

Figure 17: Mean wind pressure coefficients of the lower surfaces of the models. (a) Wind tunnel test results. (b) Numerical simulation results.

5.3.2. A Comparison of the Local Energy Input in a Vibration Period. The local energy input of the original section and the section with different upper central stabilizers at the upper and lower surface areas in a vibration cycle is given in Figures 19 and 20, respectively. For the upstream area of the upper model surface, most regions dissipate the energy of the sectional model with upper central stabilizers for heights of $0.15 \mathrm{H}, 0.25 \mathrm{H}, 0.30 \mathrm{H}$, and $0.40 \mathrm{H}$. However, most regions input energy in situations where the sectional model has upper central stabilizers with heights of $0.20 \mathrm{H}$ and $0.35 \mathrm{H}$. For the downstream region of the upper model surface, at a location of $0.0<X_{P}<0.2$, the section with central stabilizers of $0.15 \mathrm{H}, 0.25 \mathrm{H}$, and $0.35 \mathrm{H}$ inputs more energy during a vibration period, and the other sections dissipate energy or input less energy in the downstream region. Considering both upstream and downstream regions, the sum of all local work during a vibration period is negative for the sections with central stabilizers of $0.15 \mathrm{H}, 0.30 \mathrm{H}$, and $0.40 \mathrm{H}$. 


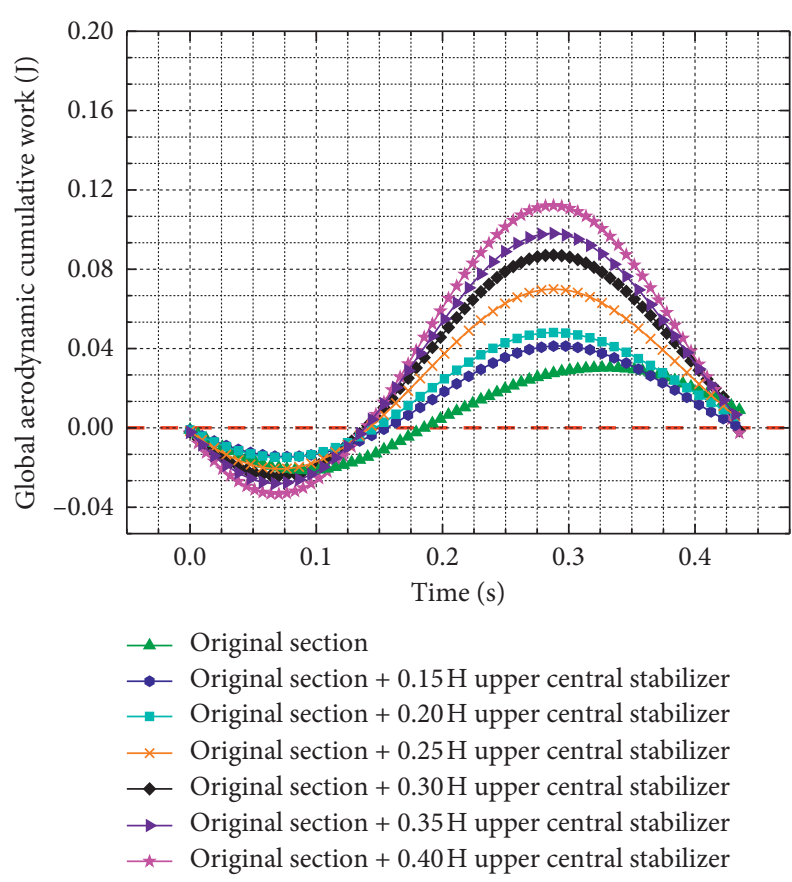

Figure 18: Global aerodynamic cumulative work for each time step.

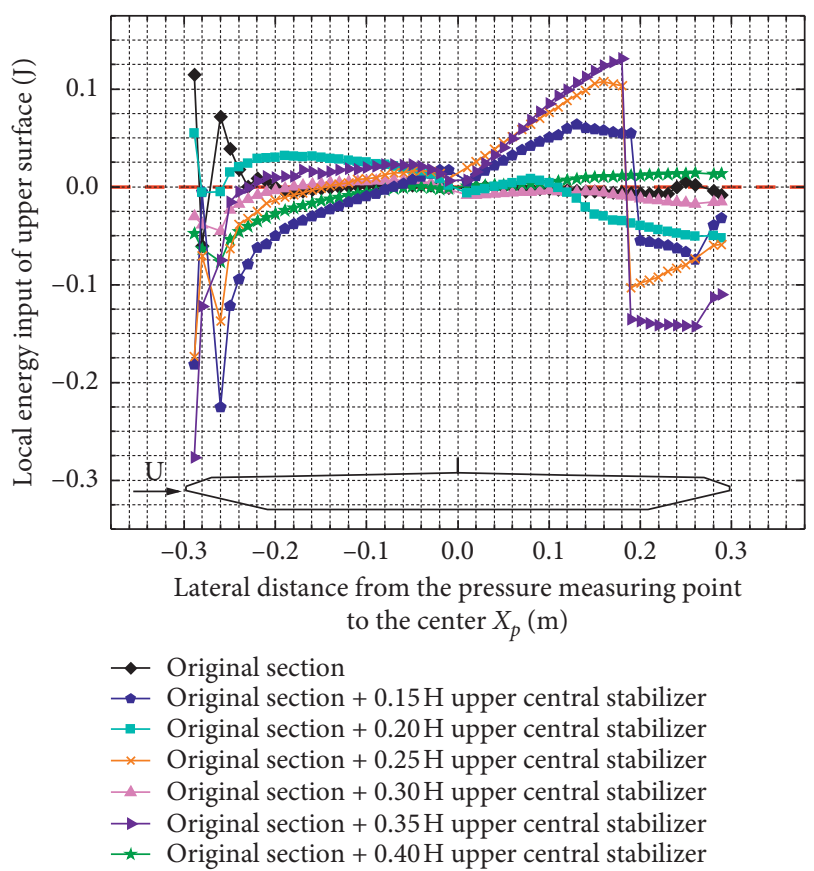

Figure 19: The local energy input of the upper surface.

Moreover, for the lower model surface of these three cases, the sum of all local work is also negative. Therefore, these results are consistent with those in Section 5.3.1, and the central stabilizers with heights of $0.15 \mathrm{H}, 0.30 \mathrm{H}$, and $0.40 \mathrm{H}$ are effective.

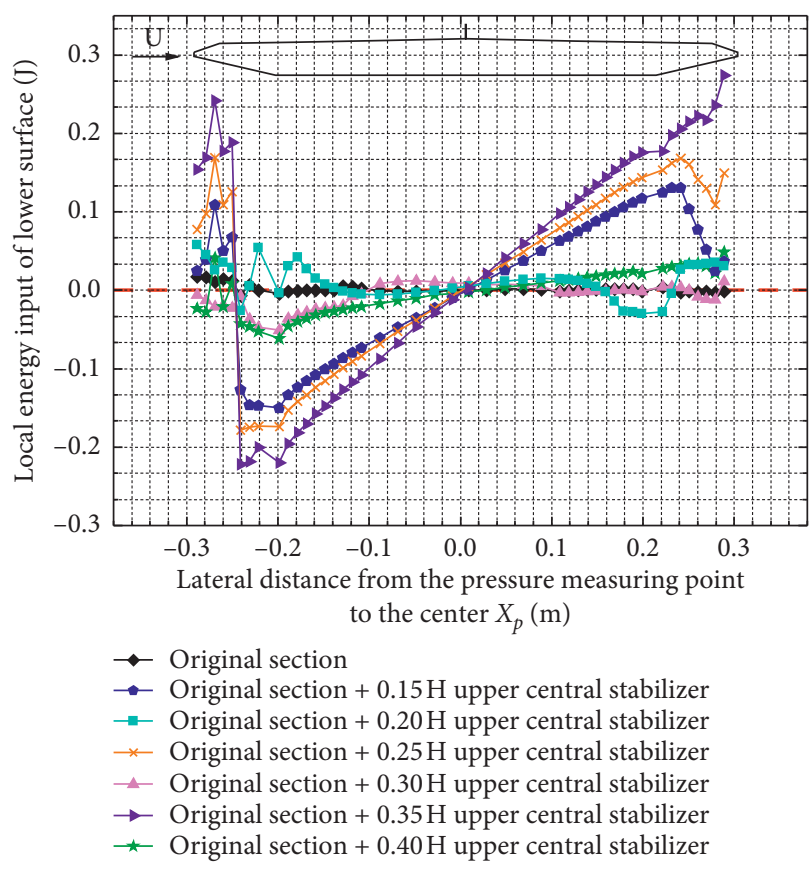

Figure 20: The local energy input of the lower surface.

\section{Concluding Remarks}

The selection and optimization of flutter aerodynamic measures are difficult to accomplish in wind tunnel tests; based on the angle of the energy exchanged in the microvibration process, both experimental and numerical methods are studied. The global and the local energy input characteristics from a macroscopic perspective were also defined. Furthermore, the mechanisms of the effectiveness of different upper central stabilizers for coupled bending and torsional flutter were studied. Moreover, according to the energy input calculation method proposed in this study, a numerical judgment of the effectiveness of the aerodynamic measures of a box girder section was discussed. The purpose of this study was to optimize aerodynamic measures through numerical analysis when the flutter of a bridge occurs in a wind tunnel test, which could provide a reference for wind tunnel tests and improve the efficiency of the test process. The main conclusions are as follows:

(1) By calculating the global energy input characteristics, the cumulative total work of the model in a complete vibration cycle can be obtained. When the cumulative total work is negative, the global aerodynamic force dissipates energy in the vibration, and the aerodynamic measures can be judged to be effective. The local energy characteristics could explain the global energy input characteristics from a microscopic perspective, thereby providing a basis for optimizing aerodynamic measures.

(2) The vibration suppression mechanism of the upper central stabilizer is such that a proper upper central 
stabilizer may produce cumulative work in the upstream region of the upper surface of the girder. The aerodynamic force in this region plays a role in dissipating energy in the vibration system. Thus, this region is a "critical region" for flutter.

(3) The numerical judgment method for the effectiveness of the upper central stabilizer has a certain reference value. When the upper central stabilizer is tested in a wind tunnel test, the recommended measures can first be obtained by numerical study, which may save time and facilitate the process.

\section{Data Availability}

The data used to support the findings of this study are available from the corresponding author upon request.

\section{Conflicts of Interest}

The authors declare that there are no conflicts of interest regarding the publication of this paper.

\section{Acknowledgments}

This research was funded by the National Natural Science Foundation of China (Grant nos. 51808053 and 51978077), the Natural Science Foundation of Shaanxi Province (Grant nos. 2018JQ5163 and 2020JM-241), and Fundamental Research Funds for the Central Universities, Chang'an University (Grant no. 300102210212).

\section{References}

[1] Z.-S. Chen, S.-M. Liu, X.-F. Yu, C.-M. Ma, and L. Liu, "Experimental investigations on VIV of bridge deck sections: a case study," KSCE Journal of Civil Engineering, vol. 21, no. 7, pp. 2821-2827, 2017.

[2] Z.-S. Chen, C. Zhang, X. Wang, and C.-M. Ma, "Wind tunnel measurements for flutter of a long-afterbody bridge deck," Sensors, vol. 17, no. 2, p. 335, 2017.

[3] G. Gao, L. Zhu, F. Wang, H. Bai, and J. Hao, "Experimental investigation on the nonlinear coupled flutter motion of a typical flat closed-box bridge deck," Sensors, vol. 20, no. 2, p. 568, 2020.

[4] Y. Yang, Y. Ge, and H. Xiang, "Flutter mechanism of representative sections for long-span bridges," Journal of Tongji University, vol. 34, no. 4, pp. 455-460, 2006, in Chinese.

[5] Y. Yang, R. Zhou, Y. Ge, X. Zou, and L. Zhang, "Flutter characteristics of twin-box girder bridges with vertical central stabilizers," Engineering Structures, vol. 133, pp. 33-48, 2017.

[6] Y. Yang, R. Zhou, Y. Ge, Y. Du, and L. Zhang, "Sensitivity analysis of geometrical parameters on the aerodynamic performance of closed-box girder bridges," Sensors, vol. 18, no. 7, p. 2053, 2018.

[7] A. Iranzo, "CFD applications in energy engineering research and simulation: an introduction to published reviews," Processes, vol. 7, no. 12, p. 883, 2019.

[8] C. A. Baxevanou, P. K. Chaviaropoulos, S. G. Voutsinas, and N. S. Vlachos, "Evaluation study of a navier-stokes CFD aeroelastic model of wind turbine airfoils in classical flutter," Journal of Wind Engineering and Industrial Aerodynamics, vol. 96, no. 8-9, pp. 1425-1443, 2008.
[9] F. Nieto, J. S. Owen, D. M. Hargreaves, and S. Hernández, "Bridge deck flutter derivatives: efficient numerical evaluation exploiting their interdependence," Journal of Wind Engineering and Industrial Aerodynamics, vol. 136, pp. 138-150, 2015.

[10] X. Ying, F. Xu, M. Zhang, and Z. Zhang, "Numerical explorations of the limit cycle flutter characteristics of a bridge deck," Journal of Wind Engineering and Industrial Aerodynamics, vol. 169, pp. 30-38, 2017.

[11] G. Vairo, "A numerical model for wind loads simulation on long-span bridges," Simulation Modelling Practice and Theory, vol. 11, no. 5-6, pp. 315-351, 2003.

[12] S. Shirai and T. Ueda, "Aerodynamic simulation by CFD on flat box girder of super-long-span suspension bridge," Journal of Wind Engineering and Industrial Aerodynamics, vol. 91, no. 1-2, pp. 279-290, 2003.

[13] M. W. Sarwar, T. Ishihara, K. Shimada, Y. Yamasaki, and T. Ikeda, "Prediction of aerodynamic characteristics of a box girder bridge section using the les turbulence model," Journal of Wind Engineering and Industrial Aerodynamics, vol. 96, no. 10-11, pp. 1895-1911, 2008.

[14] D. Sun, J. S. Owen, and N. G. Wright, "Application of the $k-\omega$ turbulence model for a wind-induced vibration study of $2 \mathrm{D}$ bluff bodies," Journal of Wind Engineering and Industrial Aerodynamics, vol. 97, no. 2, pp. 77-87, 2009.

[15] Y. Bai, D. Sun, and J. Lin, "Three dimensional numerical simulations of long-span bridge aerodynamics, using blockiterative coupling and DES," Computers \& Fluids, vol. 39, no. 9, pp. 1549-1561, 2010.

[16] A. Šarkić, R. Fisch, R. Höffer, and K.-U. Bletzinger, "Bridge flutter derivatives based on computed, validated pressure fields," Journal of Wind Engineering \& Industrial Aerodynamics, vol. 104, pp. 141-151, 2012.

[17] R. H. Scanlan, "The action of flexible bridges under wind, I: flutter theory," Journal of Sound and Vibration, vol. 60, no. 2, pp. 187-199, 1978.

[18] A. Larsen, "Aerodynamics of the tacoma narrows bridge-60 years later," Structural Engineering International, vol. 10, no. 4, pp. 243-248, 2000.

[19] X. Chen, M. Matsumoto, and A. Kareem, "Aerodynamic coupling effects on flutter and buffeting of bridges," Journal of Engineering Mechanics, vol. 126, no. 1, pp. 17-26, 2000.

[20] G. Liu, X. Wang, and S. Qiang, "Flutter analysis of long-span suspension bridges by energy method," China Journal of Highway and Transport, vol. 13, no. 3, p. 20, 2000, in Chinese.

[21] Z. Liu, Y. Yang, Y. Ge, and W. Zhang, "Numerical simulation of box-girders flutter and pneumatic energy analysis," Engineering Mechanics, vol. 32, no. 9, pp. 58-67, 2015.

[22] Y. Yang, R. Zhou, Y. Ge, and L. Zhang, "Flutter characteristics of thin plate sections for aerodynamic bridges," Journal of Bridge Engineering, vol. 23, no. 1, 2018.

[23] H. Tang, Y. Li, Y. Wang, and Q. Tao, "Aerodynamic optimization for flutter performance of steel truss stiffening girder at large angles of attack," Journal of Wind Engineering and Industrial Aerodynamics, vol. 168, pp. 260-270, 2017. 